\title{
Stool microbiota composition is associated with the prospective risk of Plasmodium falciparum infection
}

\author{
Shibu Yooseph ${ }^{1}$, Ewen F. Kirkness' ${ }^{2}$, Tuan M. Tran³ ${ }^{3}$ Derek M. Harkins², Marcus B. Jones ${ }^{1}$, Manolito G. Torralba ${ }^{2}$, \\ Elise O'Connell ${ }^{4}$, Thomas B. Nutmann ${ }^{4}$, Safiatou Doumbo ${ }^{5}$, Ogobara K. Doumbo ${ }^{5}$, Boubacar Traore ${ }^{5}$, \\ Peter D. Crompton ${ }^{3^{*}}$ and Karen E. Nelson ${ }^{1 *}$
}

\begin{abstract}
Background: In humans it is unknown if the composition of the gut microbiota alters the risk of Plasmodium falciparum infection or the risk of developing febrile malaria once $P$. falciparum infection is established. Here we collected stool samples from a cohort composed of 195 Malian children and adults just prior to an intense $P$. falciparum transmission season. We assayed these samples using massively parallel sequencing of the $16 \mathrm{~S}$ ribosomal RNA gene to identify the composition of the gut bacterial communities in these individuals. During the ensuing 6-month $P$. falciparum transmission season we examined the relationship between the stool microbiota composition of individuals in this cohort and their prospective risk of both $P$. falciparum infection and febrile malaria.

Results: Consistent with prior studies, stool microbial diversity in the present cohort increased with age, although the overall microbiota profile was distinct from cohorts in other regions of Africa, Asia and North America. Age-adjusted Cox regression analysis revealed a significant association between microbiota composition and the prospective risk of P. falciparum infection; however, no relationship was observed between microbiota composition and the risk of developing febrile malaria once $P$. falciparum infection was established.

Conclusions: These findings underscore the diversity of gut microbiota across geographic regions, and suggest that strategic modulation of gut microbiota composition could decrease the risk of $P$. falciparum infection in malaria-endemic areas, potentially as an adjunct to partially effective malaria vaccines.
\end{abstract}

Keywords: Stool microbiota, Gut microbiota, Malaria, 16S rRNA gene sequencing, Plasmodium falciparum, Human, Prospective cohort

\section{Background}

The human microbiome represents the trillions of microbes that naturally inhabit the human body, the vast majority of which reside within the gastrointestinal tract [1]. Major advances in sequencing technology have allowed for investigations of the microbiome in the context of health and disease. Large-scale studies of the westernized human microbiome, including the NIH funded Roadmap Human Microbiome Project (HMP) $[2,3]$

\footnotetext{
* Correspondence: pcrompton@niaid.nih.gov; kenelson@jcvi.org ${ }^{3}$ Laboratory of Immunogenetics, Division of Intramural Research, National Institute of Allergy and Infectious Diseases, National Institutes of Health, Rockville, MD 20852, USA

'J. Craig Venter Institute, 4120 Capricorn Lane, La Jolla, CA 92037, USA Full list of author information is available at the end of the article
}

targeting several body sites and the European Commission funded Metagenomics of the Human Intestinal Tract [4], have defined the healthy human microbiome, providing a reference for subsequent studies into the interactions between microbes and their environment in the context of host immunity and/or genetics.

Systematic studies aimed at understanding the complex interactions between the host and microbiota have demonstrated that the composition of the gut microbiota varies with age [5-7], race/ethnicity [2], geographical location $[5,6]$, and dietary intake [7-9]. The role of the microbiota in modulating host immune responses to infectious pathogens is also increasingly recognized [10]. Indeed, the composition of the gut microbiota has been 
shown to influence both local [11-13] and systemic [14-16] immune responses.

Among parasitic pathogens, Plasmodium falciparum malaria, which is transmitted by Anopheles mosquitos, imposes the greatest disease burden worldwide. The World Health Organization (WHO) estimates that 3.3 billion people are at risk of malaria in 97 countries. In 2013, there were approximately 198 million cases of malaria and an estimated 584,000 deaths, $90 \%$ of which occurred in Africa, with children under 5 years accounting for $78 \%$ of all deaths [17].

In areas of intense malaria transmission, immunity to life-threatening malaria is typically acquired by early childhood, whereas older children remain susceptible to repeated bouts of febrile malaria through adolescence, eventually acquiring near-complete immunity to the disease caused by blood-stage malaria parasites by early adulthood [18, 19]. However, despite repeated infections over years, sterilizing acquired immunity to $P$. falciparum infection seems to occur rarely [20]. The nature of the human immune response that protects from P. falciparum infection and the disease it causes is complex and only partially understood [21]. Indeed, the quality of the immune response and the clinical outcome during any given $P$. falciparum infection can vary greatly between and within individuals [22]. Although host and parasite genetic factors $[23,24]$ clearly contribute to the heterogeneity in immune and clinical responses to malaria, it is likely that environmental factors such as the host microbiota also play a role. For example, a recent mouse study demonstrated that anti- $\alpha$-gal antibodies-induced by the presence of Escherichia coli O86:B7 in the gut-are cytotoxic to $\alpha-$ gal-expressing Plasmodium sporozoites, and thus protect mice from mosquito-transmitted Plasmodium infection [25]. In the same report, an association was observed between anti- $\alpha$ gal IgM levels and protection from P. falciparum infection in Malian children and adults residing in an area of intense malaria transmission [25], suggesting that the host microbiota may play a role in susceptibility to $P$. falciparum infection in humans. In addition, Li et al. [26] noted that experimental infection of mice with Plasmodium berghei resulted in altered metabolite profiles that they attributed to a disturbance of the gut microbial community caused by the parasite. However, it is currently unknown in humans if the composition of the gut microbiota modulates the risk of $P$. falciparum infection or the risk of developing malarial disease once $P$. falciparum blood-stage infection is established.

To investigate the potential interplay between $P$. falciparum and the gut microbiota, we analyzed the bacterial communities in stool samples collected from a cohort of 195 Malian children and adults just prior to an intense malaria transmission season. Community composition was determined by sequencing the $16 \mathrm{~S}$ ribosomal
RNA (rRNA) gene using high-throughput next generation sequencing technology. In a prospective cohort study we examined the relationship between the gut microbiota composition of these individuals and their subsequent risk of $P$. falciparum infection and febrile malaria. We also compared the microbiota composition of this cohort with published studies conducted in other regions. Consistent with prior studies, we found that gut microbial diversity in this cohort increased with age, although the overall microbiota profile was distinct from cohorts in other regions of Africa, Asia and North America. Age-adjusted Cox regression analysis revealed a significant association between the microbiota composition before the malaria season and the prospective risk of $P$. falciparum infection during the ensuing malaria season, while no relationship was observed between microbiota composition and the risk of developing febrile malaria once $P$. falciparum infection was established.

\section{Results}

\section{Characteristics of study subjects}

From May 11 - May 31, 2011 (end of 6-month dry season) we enrolled 695 healthy individuals in this cohort study. Stool was collected from all subjects at the time of enrollment. A subset of 200 individuals was randomly selected in an age-stratified manner for stool microbiota analysis (Fig. 1). Of these 200 subjects, 55 subjects $(28 \%)$ were asymptomatically infected with $P$. falciparum blood-stage parasites by PCR at enrollment when stool was also collected. The prevalence of intestinal helminths at enrollment was near zero, consistent with the mass-drug treatment program in this region of Mali, whereas the prevalence of Schistosoma haematobium in the urine was $7.4 \%$, mostly among adolescents and adults. The prevalence of sickle cell trait (HbAS) was $8.5 \%$. Detailed demographic and clinical data stratified by age are shown in Table 1 . Consistent with our findings in the entire cohort [20], longitudinal analysis during the ensuing malaria season showed that the risk of febrile malaria decreased with increasing age in the subset of individuals in this study, with the exception of infants under the age of 1 year whose risk was intermediate between children and adults (Table 2), presumably due to the protective effect of maternal antibodies [27].

\section{Diversity and composition of the microbiota in Mali stool samples}

Sequence data were successfully generated from 195 out of the 200 stool samples. After processing the raw sequence data, the combined dataset consisted of 1,331,167 sequences. However, not all sequences could be confidently assigned taxonomy to the genus level; therefore we denote these sequences by appending the tag unclassified to the end of their taxonomic assignment (one of phylum, class, order, or family levels). The taxonomic assignments 


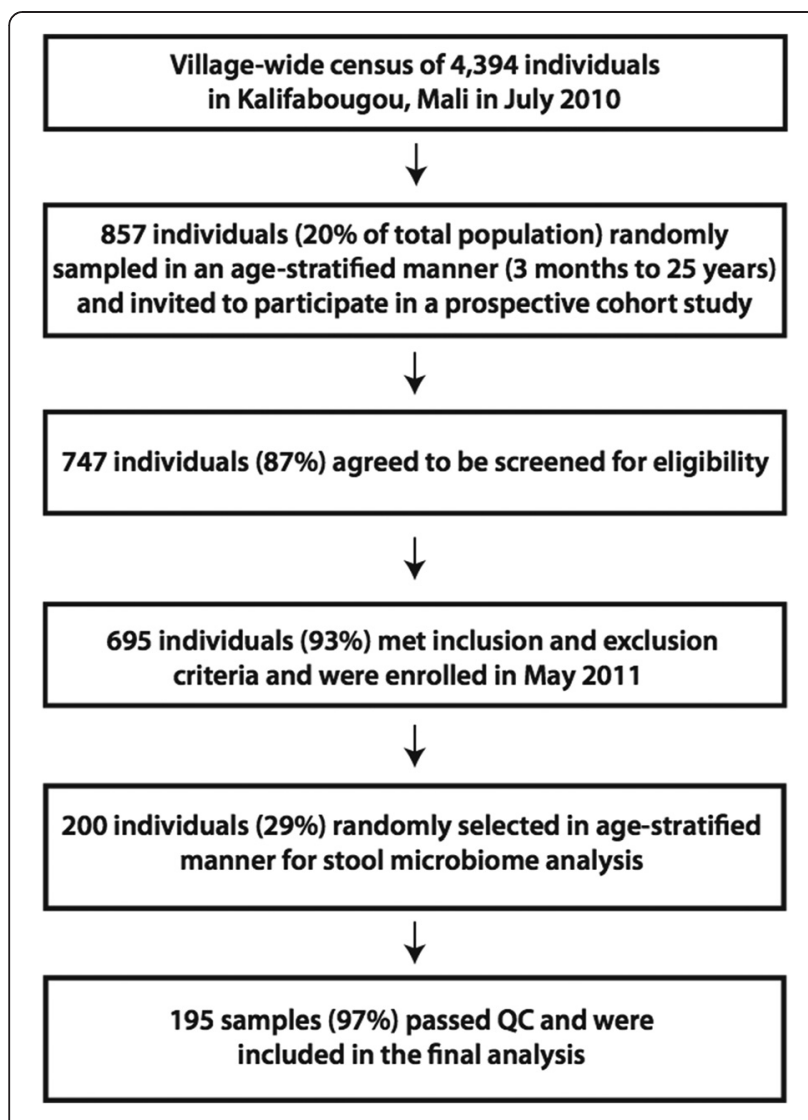

Fig. 1 Selection of study subjects for stool microbiota analysis

were used to generate a sample-taxa count matrix. In the combined dataset, Bifidobacterium, Streptococcus, and family Ruminococcaceae unclassified were the three most abundant taxonomic groups, accounting for $13.7 \%$, $12.9 \%$, and $12.4 \%$ of sequences, respectively.

Operational Taxonomic Units (OTUs) were identified in these data by clustering sequences at $97 \%$ sequence identity, and were used to compute microbial community diversity (alpha diversity) in these samples. Subsequently, the relationship between diversity and age was assessed using a linear model. This analysis revealed that diversity increased with age in this cohort (Fig. 2); a similar trend was observed for both genders separately (Additional file 1: Figure S1).

The sample-taxa count matrix was further analyzed using a Dirichlet Multinomial Mixture (DMM) modeling framework [28] to identify sample groups (or Dirichlet components). An evaluation of the model fit (Additional file 1: Figure S2) revealed that two Dirichlet components (hereafter referred to as DC1 and DC2) provided the best fit for these data. Figure 3 shows a two-dimensional ordination of these samples that was generated using non-metric multidimensional scaling (NMDS) based on Bray-Curtis dissimilarity between samples. The individuals comprising the sample groups $\mathrm{DC} 1(n=133)$ and
DC2 $(n=62)$ were significantly different in terms of age $\left(P<10^{-15}\right.$; assessed using a $t$-test $)$ and taxonomic profiles $(P \leq 0.05$; assessed by non-overlapping $95 \%$ credible intervals), but had similar gender distributions. Specifically, the average ages of individuals in DC1 and DC2 were 10.3 years and 1.3 years, respectively. The most abundant taxa in the older DC1 group were family Ruminococcaceae unclassified (8.7 \%), family Lachnospiraceae unclassified (8.5 \%), and Faecalibacterium (7.4 \%), while the most abundant taxa in the younger DC2 group were Bifidobacterium (17.1\%) and Streptococcus (11.6 \%) (Fig. 4 and Additional file 1: Table S1).

\section{Comparison of Mali, Malawi, HMP, and MSD cohorts}

Next, we compared the gut microbiota composition of the Mali cohort with data from the Malawi, HMP, and MSD microbiome studies described in Material and Methods. The four datasets were analyzed based on taxonomies of their constituent samples. DMM modeling identified 9 Dirichlet components (CC1 - CC9) (Additional file 1: Table S2), each with a different taxonomic profile (Additional file 1: Table S3). The 9 Dirichlet sample groups largely fell along cohort lines (Additional file 1: Table S4). For example, the Dirichlet components DC1 and DC2 that were identified by DMM modeling of the Mali cohort were largely preserved as components CC2 and CC5, respectively. Like CC5, which was comprised exclusively of Mali samples, CC6 had a high abundance of Bifidobacterium and Streptococcus, but only contained samples from the Malawi cohort; however, the proportions of Bifidobacterium and Streptococcus were different in CC6 ( $22 \%$ and $5 \%$, respectively) compared to CC5 (15\% and $11 \%$, respectively). The HMP samples were predominantly split across CC4 and CC9, both of which were dominated by Bacteroides, but at different abundance levels ( $25 \%$ and $60 \%$, respectively). The MSD cohort was mostly split across $\mathrm{CC} 1, \mathrm{CC} 3$, and $\mathrm{CC} 8$, all of which were dominated by Prevotella to varying degrees (8\% in $\mathrm{CC} 1$ and $70 \%$ in $\mathrm{CC} 3$ ) and family Enterobacteriaceae unclassified (9.6 \% in CC8). CC7 consisted of Malawi samples and was also dominated by Prevotella (21\%).

\section{Relationship between gut microbiota and persistent}

$P$. falciparum infection, incident $P$. falciparum infection, and incident febrile malaria in the Malian cohort

We investigated the relationship between the gut microbiota composition and three distinct clinical phenotypes in the Malian cohort: persistent P. falciparum infection, incident $P$. falciparum infection, and incident febrile malaria. These three phenotypes are described in Fig. 5.

In a cross-sectional analysis at the end of the 6-month dry season, a period of negligible malaria transmission [29], we first compared the gut microbiota composition of individuals with persistent, asymptomatic $P$. falciparum 
Table 1 Baseline characteristics of study subjects by age group

\begin{tabular}{|c|c|c|c|c|c|c|c|}
\hline \multirow[t]{2}{*}{ Characteristic } & \multicolumn{6}{|l|}{ Age Group } & \multirow[t]{2}{*}{ All $(n=200)$} \\
\hline & $\begin{array}{l}3-5 \text { mo } \\
(n=26)\end{array}$ & $\begin{array}{l}6 \text { mo-1 y } \\
(n=41)\end{array}$ & $\begin{array}{l}2-5 y \\
(n=37)\end{array}$ & $\begin{array}{l}6-11 \text { y } \\
(n=44)\end{array}$ & $\begin{array}{l}12-17 y \\
(n=24)\end{array}$ & $\begin{array}{l}18-25 y \\
(n=28)\end{array}$ & \\
\hline Female gender & $11(42 \%)$ & $26(63 \%)$ & $16(43 \%)$ & $23(52 \%)$ & $11(46 \%)$ & $19(68 \%)$ & $106(53 \%)$ \\
\hline Bambara ethnicity & $21(81 \%)$ & 37 (90 \%) & $32(86 \%)$ & $38(86 \%)$ & $22(92 \%)$ & $24(86 \%)$ & $174(87 \%)$ \\
\hline $\begin{array}{l}\text { P. falciparum } \\
\text { PCR positive } \\
\text { at enrollment }\end{array}$ & $0(0 \%)$ & $10(24 \%)$ & 11 (30 \%) & $4(9.1 \%)$ & $16(67 \%)$ & $14(50 \%)$ & $55(28 \%)$ \\
\hline $\begin{array}{l}\text { P. malariae } \\
\text { PCR positive } \\
\text { at enrollment }\end{array}$ & 0 & 0 & 0 & 0 & 1 (4.2\%) & 0 & $1(0.5 \%)$ \\
\hline \multicolumn{8}{|c|}{$\begin{array}{l}\text { Stool PCR for intestinal helminths } \\
\text { (number positive/number tested) }\end{array}$} \\
\hline A. duodenale & & & & $0 / 15$ & $0 / 24$ & $0 / 28$ & $0 / 67$ \\
\hline A. lumbricoides & & & & $0 / 11$ & $0 / 24$ & $0 / 28$ & $0 / 63$ \\
\hline N. americanus & & & & $0 / 22$ & $0 / 24$ & $1 / 28(3.6 \%)$ & $1 / 74(1.4 \%)$ \\
\hline S. stercoralis & & & & $0 / 11$ & $0 / 24$ & $0 / 28$ & $0 / 63$ \\
\hline T. trichura & & & & $0 / 11$ & $0 / 24$ & $0 / 28$ & $0 / 63$ \\
\hline \multicolumn{8}{|c|}{$\begin{array}{l}\text { Kato-Katz for intestinal and urinary helminths } \\
\text { (number positive/number tested) }\end{array}$} \\
\hline S. mansoni (stool) & $0 / 24$ & $0 / 40$ & $0 / 37$ & $0 / 44$ & $0 / 24$ & $0 / 28$ & 0/197 \\
\hline H. nana (stool) & $0 / 24$ & $0 / 40$ & $0 / 37$ & $0 / 44$ & $0 / 24$ & $0 / 28$ & 0/197 \\
\hline $\begin{array}{l}\text { S.haematobium } \\
\text { (urine) }\end{array}$ & 2/19 (11\%) & $1 / 39(2.6 \%)$ & 0/37 (0 \%) & $1 / 43(2.3 \%)$ & $2 / 16(12 \%)$ & $7 / 22(32 \%)$ & 13/176 (7.4\%) \\
\hline \multicolumn{8}{|l|}{ Hb type } \\
\hline $\mathrm{AA}$ & $23(88 \%)$ & $35(85 \%)$ & $29(78 \%)$ & $38(86 \%)$ & $22(92 \%)$ & 19 (68 \%) & $166(83 \%)$ \\
\hline$A C$ & 1 (3.8 \%) & $3(7.3 \%)$ & $6(16 \%)$ & 2 (4.5 \%) & 1 (4.2\%) & $3(11 \%)$ & 16 (8 \%) \\
\hline AS & 2 (7.7 \%) & $3(7.3 \%)$ & 2 (5.4 \%) & 4 (9.1\%) & 1 (4.2\%) & 5 (18\%) & 17 (8.5 \%) \\
\hline SC & 0 & 0 & 0 & 0 & 0 & 1 (3.6\%) & $1(0.5 \%)$ \\
\hline $\begin{array}{l}\text { Hemoglobin, } \\
\text { g/dL (95 \% Cl) }\end{array}$ & $11.0(10.7-11.3)$ & $10.2(9.9-10.6)$ & $11.1(10.8-11.5)$ & $12.3(12.0-12.6)$ & $13.0(12.6-13.4)$ & $13.5(12.9-14.0)$ & $11.7(11.5-12.0)$ \\
\hline
\end{tabular}

${ }^{\mathrm{a}}$ Before the malaria season and at time of stool collection. All $P$. falciparum-infected subjects at the time of enrollment were asymptomatic

blood-stage infection versus uninfected individuals. P. falciparum infections detected at the end of the 6-month dry season are generally acquired during the preceding transmission season and persist through the dry season at low levels without causing symptoms. This analysis was carried out using the DMM framework, but in a supervised manner with subjects classified as either persistently infected $(n=55)$ or uninfected $(n=140)$. An NMDS ordination of the samples along with their $P$. falciparum infection status at the end of the dry season is shown in Fig. 6. Among the 55 persistently infected subjects we identified two Dirichlet components PP1 (15 subjects) and PP2 (40 subjects), with average subject ages of 3.2 years and 13.6 years, respectively (Additional file 1: Table S5). Among the 140 uninfected subjects we identified two components PN1 (56 subjects) and PN2 (84 subjects) with average subject ages of 1.3 years and 9.2 years, respectively (Additional file 1: Table S5). The mean vector of taxa proportions for component PP2 (older and infected) is very close to that of component PN2 (older and uninfected) indicating that these two subject groups have very similar taxonomic proportions, with no statistically significant differences (Fig. 7, Additional file 1: Table S6). The mean of component PP1 (young and infected) was not as close to the means of either of PN1 or PN2. However, the only taxa in this component that had a significantly different proportion compared to its values in $\mathrm{PN} 1$ and $\mathrm{PN} 2$ was Bifidobacterium (3.6\%). Together, these findings suggest that age may be a stronger predictor of gut microbiota composition than $P$. falciparum infection status at the end of the 6-month dry season (i.e. persistent $P$. falciparum infection versus no infection).

Next, we examined the relationship between the stool microbiota composition present before the malaria season and the prospective risk of becoming infected with $P$. falciparum blood-stage parasites during the ensuing 6-month malaria season. For this analysis we only included subjects who were not infected with $P$. falciparum (by PCR) before 
Table 2 Malaria outcomes by age group

\begin{tabular}{|c|c|c|c|c|c|c|c|}
\hline \multirow[t]{2}{*}{ Outcome } & \multicolumn{6}{|l|}{ Age Group } & \multirow[t]{2}{*}{$P$ value } \\
\hline & $3-5 \mathrm{mo}(n=26)$ & $6 \mathrm{mo}-1$ y $(n=41)$ & $2-5$ y $(n=37)$ & $6-11$ y $(n=44)$ & $12-17$ y $(n=24)$ & $18-25$ y $(n=28)$ & \\
\hline $\begin{array}{l}\text { Median days from enrollment } \\
\text { to first malaria episode }{ }^{\mathrm{a}}(95 \% \mathrm{Cl})\end{array}$ & 227 (159-249) & $\mathrm{NE}$ & $134(109-176)$ & $142(120-257)$ & $\mathrm{NE}$ & $\mathrm{NE}$ & $<0.0001^{*}$ \\
\hline $\begin{array}{l}\text { Median parasite density for first } \\
\text { malaria episodes in parasites/ } \mu \mathrm{L} \\
\text { (interquartile range) }\end{array}$ & $\begin{array}{l}14,400 \\
(9,600-47,850)\end{array}$ & $\begin{array}{l}38,740 \\
(18,210-173,400)\end{array}$ & $\begin{array}{l}19,800 \\
(8,700-57,680)\end{array}$ & $\begin{array}{l}16,300 \\
(5,700-76,300)\end{array}$ & $\begin{array}{l}13,920 \\
(7,412-24,000)\end{array}$ & $\begin{array}{l}10,720 \\
(9,012-14,080)\end{array}$ & $0.19^{* *}$ \\
\hline $\begin{array}{l}\text { Individuals with } \geq 1 \text { malaria } \\
\text { episodes ( } \% \text { of age group) }\end{array}$ & $4(15 \%)$ & $5(12 \%)$ & $13(35 \%)$ & $13(30 \%)$ & $1(4.2 \%)$ & 0 & $<0.001^{* * *}$ \\
\hline
\end{tabular}

Abbreviations: $\mathrm{Cl}$, confidence interval; $N E$, not estimated because $>50 \%$ of individuals remained free of malaria during the study period

${ }^{a}$ Malaria defined as $\geq 2500$ parasites $/ \mu \mathrm{L}$, an axillary temperature of $\geq 37.5^{\circ} \mathrm{C}$ within $24 \mathrm{~h}$, and no other cause of fever discernible by physical exam

*log-rank test

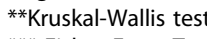

**** Fisher Exact Test 


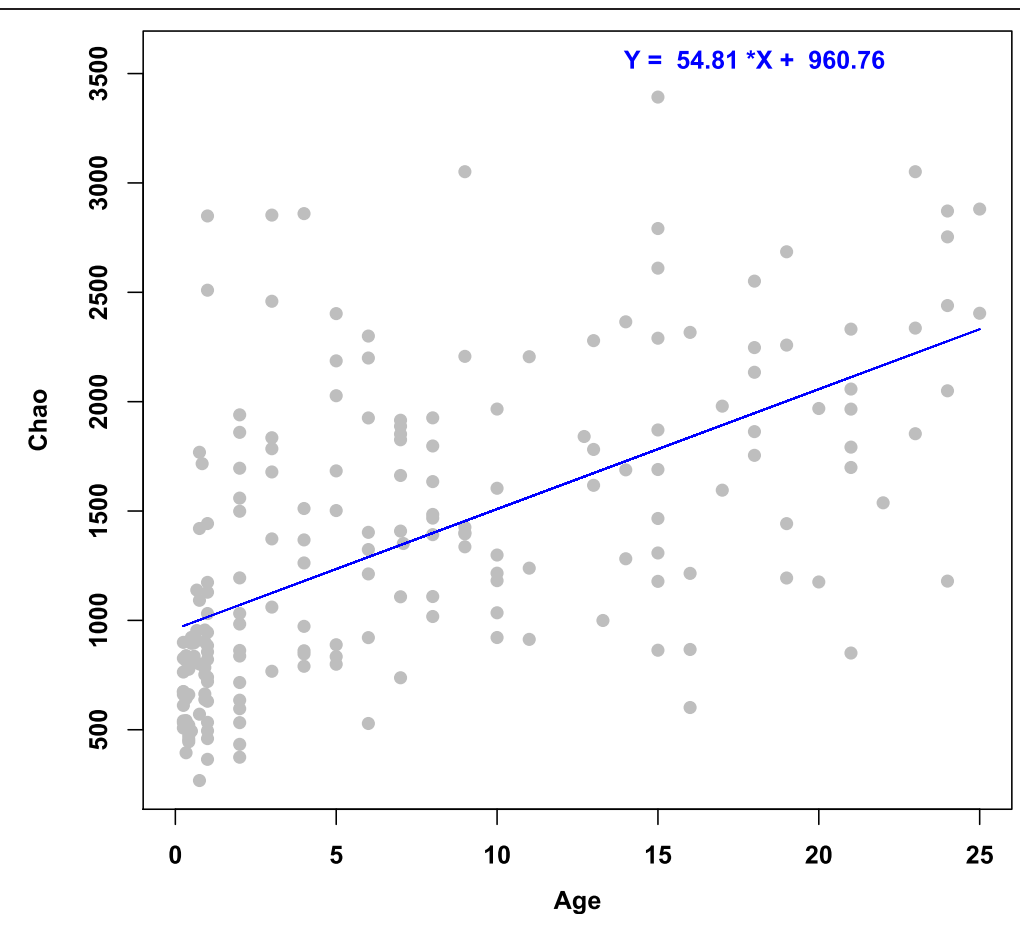

Fig. 2 Increase in microbial taxa diversity with age in the Malian cohort. For each stool microbiota sample, the alpha diversity (Chao estimate) of the microbial community (y-axis) was plotted against the individual's age in years ( $\mathrm{x}$-axis). The blue line is the linear model fit and has a positive slope $\left(P<10^{-15}\right)$

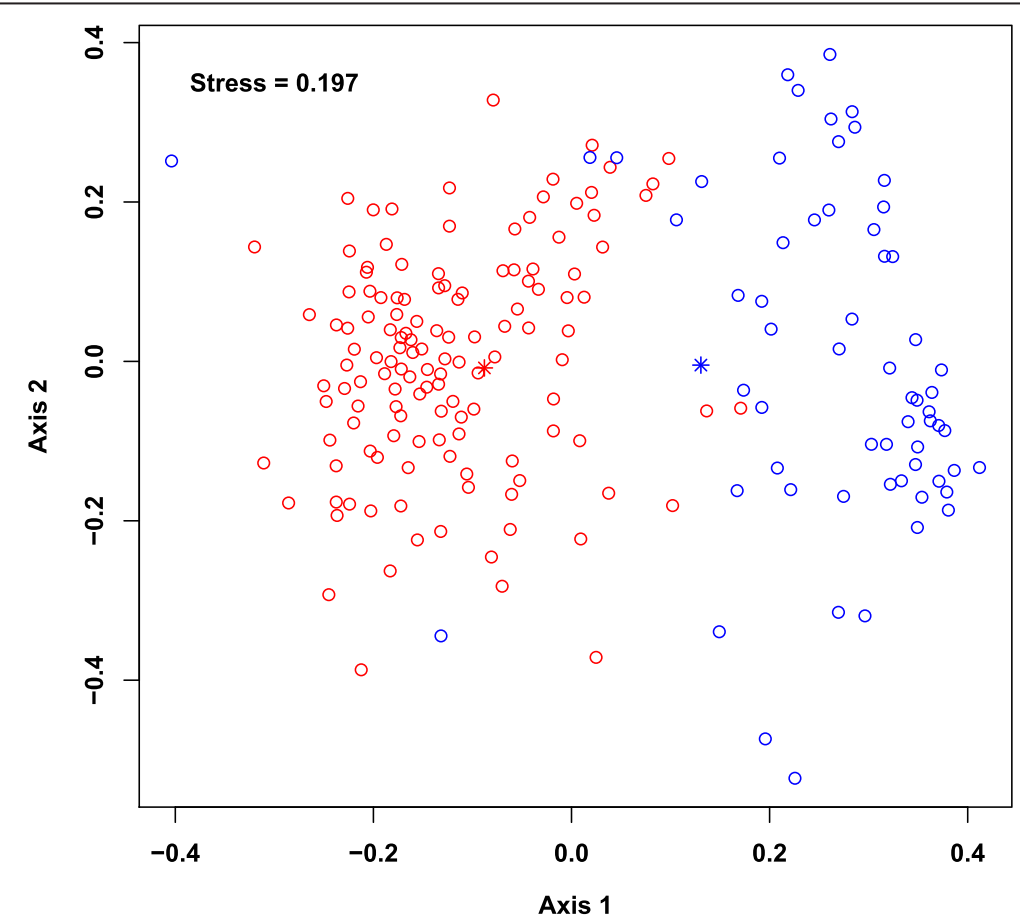

Fig. 3 An NMDS ordination of the microbiota samples. The taxonomic profiles of the samples were used to compute a sample dissimilarity matrix (using Bray-Curtis measure) and this matrix was used to compute an ordination of the samples in two dimensions (Axis 1 and Axis 2). The goodness of fit (or stress) associated with this ordination is 0.197 . The circles in this plot denote the samples. The two sample groups (or Dirichlet components) identified using the mixture modeling are denoted in red (DC1) and blue (DC2). The average ages for the groups are 10.3 years (DC1) and 1.3 years (DC2). Asterisks denote the vectors of mean taxa proportions associated with the corresponding Dirichlet components 


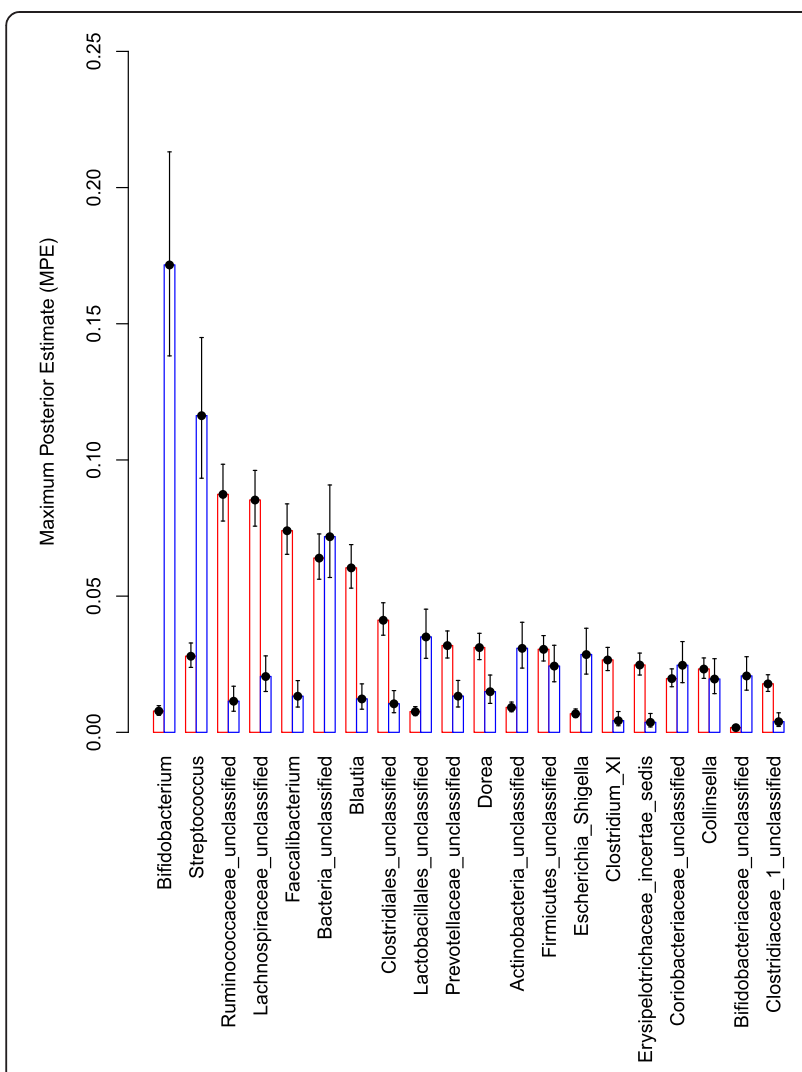

Fig. 4 Mean proportions (calculated using Maximum Posterior Estimate) of the twenty most abundant taxa (based on abundance in one of the groups) in the two Dirichlet components: the older DC1 group (red) and younger DC2 group (blue). The lower and upper $95 \%$ credible intervals are shown as error bars the malaria season and who had completed study follow-up through the end of the ensuing 6-month malaria season (112 subjects). DMM modeling of the microbiota data obtained from these $P$. falciparum uninfected subjects identified two Dirichlet components FM1 (38 subjects) and FM2 (74 subjects). These two sample groups had distinct taxonomic profiles (Additional file 1: Table S7, Fig. 8). Bifidobacterium (22\%) and Streptococcus (10\%) were the most abundant taxa in FM1 while family Ruminococcaceae unclassified (9.3\%), and family Lachnospiraceae unclassified $(9.1 \%)$ were the most abundant taxa in FM2. In addition to these taxa, other taxa including Faecalibacterium, Blautia, order Clostridiales unclassified, family Prevotellaceae unclassified, and Escherichia/Shigella were differentially abundant across the two sample groups $(\mathrm{P} \leq 0.05$; assessed by non-overlapping $95 \%$ credible intervals). The average age of subjects in FM1 and FM2 was 1.4 years and 9.1 years, respectively. During the ensuing malaria season, incident (new) $P$. falciparum blood-stage infections in these subjects were detected through bi-weekly PCR analysis of fingerprick blood samples. We compared the time to first $P$. falciparum infection in the FM1 versus FM2 sample groups (irrespective of whether the infection caused symptoms) by generating Kaplan-Meier plots and performing log-rank analysis (Fig. 9). We noted a statistically significant delay in time to first $P$. falciparum infection (Fig. 9a) for FM1 subjects (median: 121 days; $95 \%$ CI 101-150) compared to FM2 subjects (median: 85 days; $95 \% \mathrm{CI}$ 73-99; $\mathrm{P}=0.0002$ ). Moreover, Cox regression analysis accounting for age, gender, anemia, HbAS, S. haematobium infection, splenomegaly and distance to the river

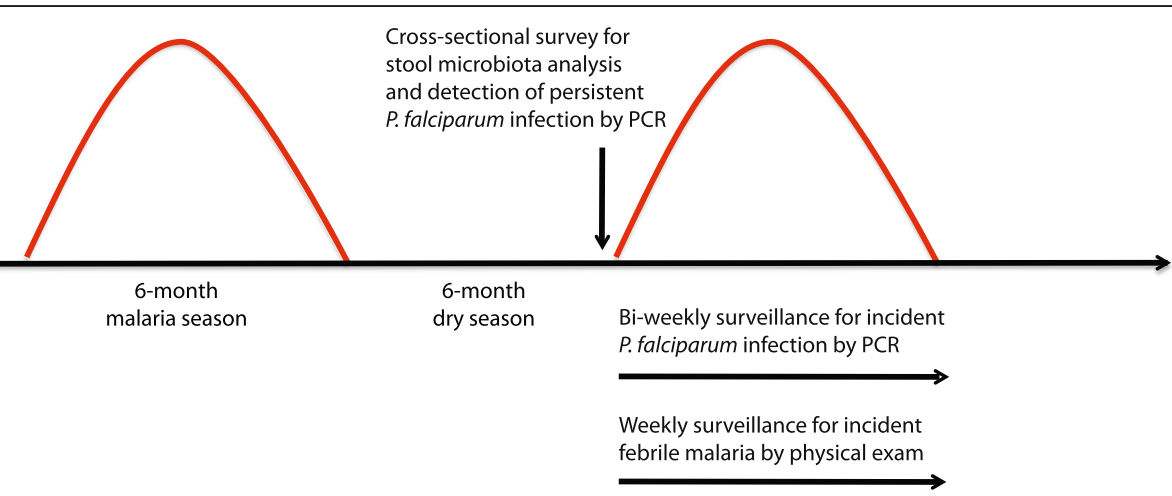

Fig. 5 Definition of persistent $P$. falciparum infection, incident $P$. falciparum infection, and incident febrile malaria. This longitudinal cohort study in Mali was designed to take advantage of the sharply demarcated 6-month malaria season (July - December) and 6-month dry season (January - June) during which there is either intense or negligible P. falciparum transmission, respectively. Stool microbiota composition was determined for all study subjects in a cross-sectional survey at the end of the 6-month dry season. For each subject, we examined the relationship between stool microbiota composition and the risk of persistent $P$. falciparum infection, incident $P$. falciparum infection and incident febrile malaria. Individuals with $P$. falciparum infections that persisted without symptoms through the dry season were identified by PCR analysis of fingerprick blood samples in a cross-sectional survey at the end of the 6-month dry season (at the time of stool collection). For subjects who began the malaria season without $P$. falciparum infection, incident P. falciparum infections during the ensuing malaria season were detected prospectively through bi-weekly PCR analysis of fingerprick blood samples. For all subjects who became infected with P. falciparum blood-stage parasites, incident cases of febrile malaria (axillary temperature $\geq 37.5^{\circ} \mathrm{C}$ and $\geq 2500$ asexual parasites $/ \mu$ of blood) were detected prospectively during the ensuing malaria season through weekly physical examination by the study physician and blood smear microscopy if malaria was suspected 


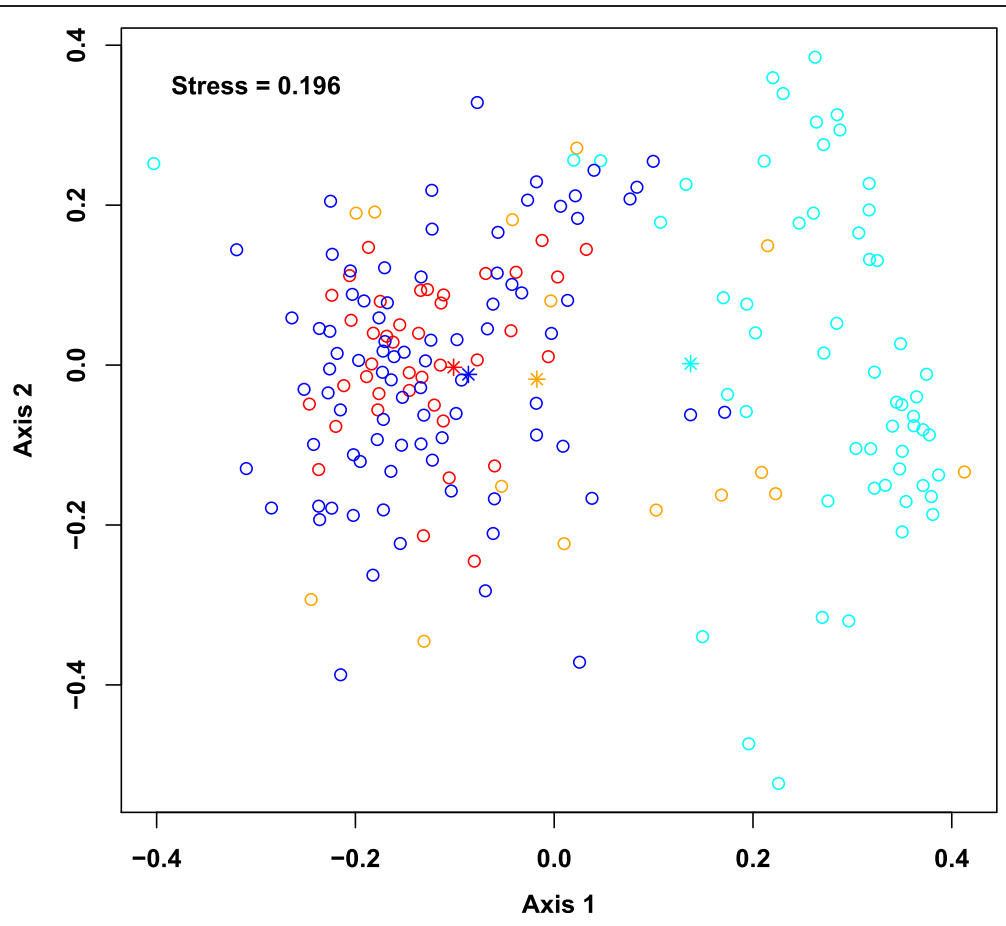

Fig. 6 NMDS ordination of the Mali samples on the basis of $P$. falciparum infection status at the end of the 6-month dry season. Samples from subjects with persistent, asymptomatic P. falciparum infection at the end of the dry season fall into two components PP1 (orange) and PP2 (red); samples from P. falciparum-uninfected subjects fall into two components as well - PN1 (cyan) and PN2 (blue). The mean vector of each component is labeled with an asterisk in their respective color. The average ages for the groups are 3.2 years (PP1), 13.6 years (PP2), 1.3 years (PN1) and 9.2 years (PN2)

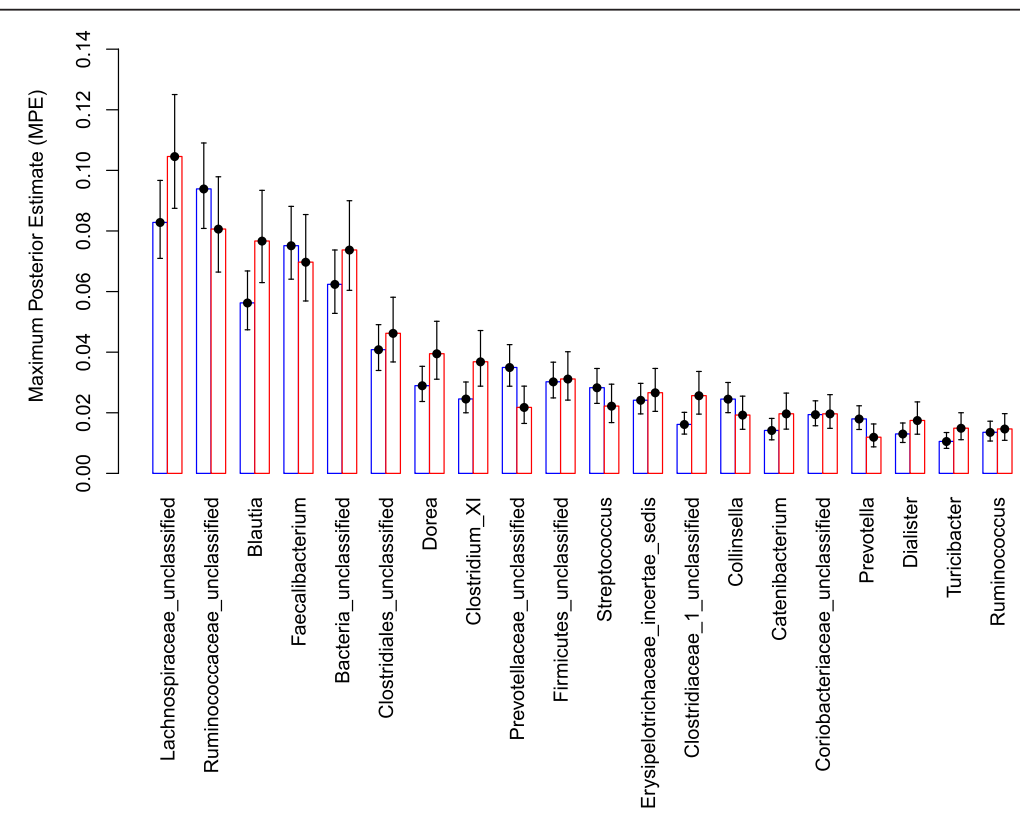

Fig. 7 Mean proportions (Maximum Posterior Estimate) of the twenty most abundant taxa in the two Dirichlet components PP2 (red) and PN2 (blue). The lower and upper $95 \%$ credible intervals are shown as error bars 


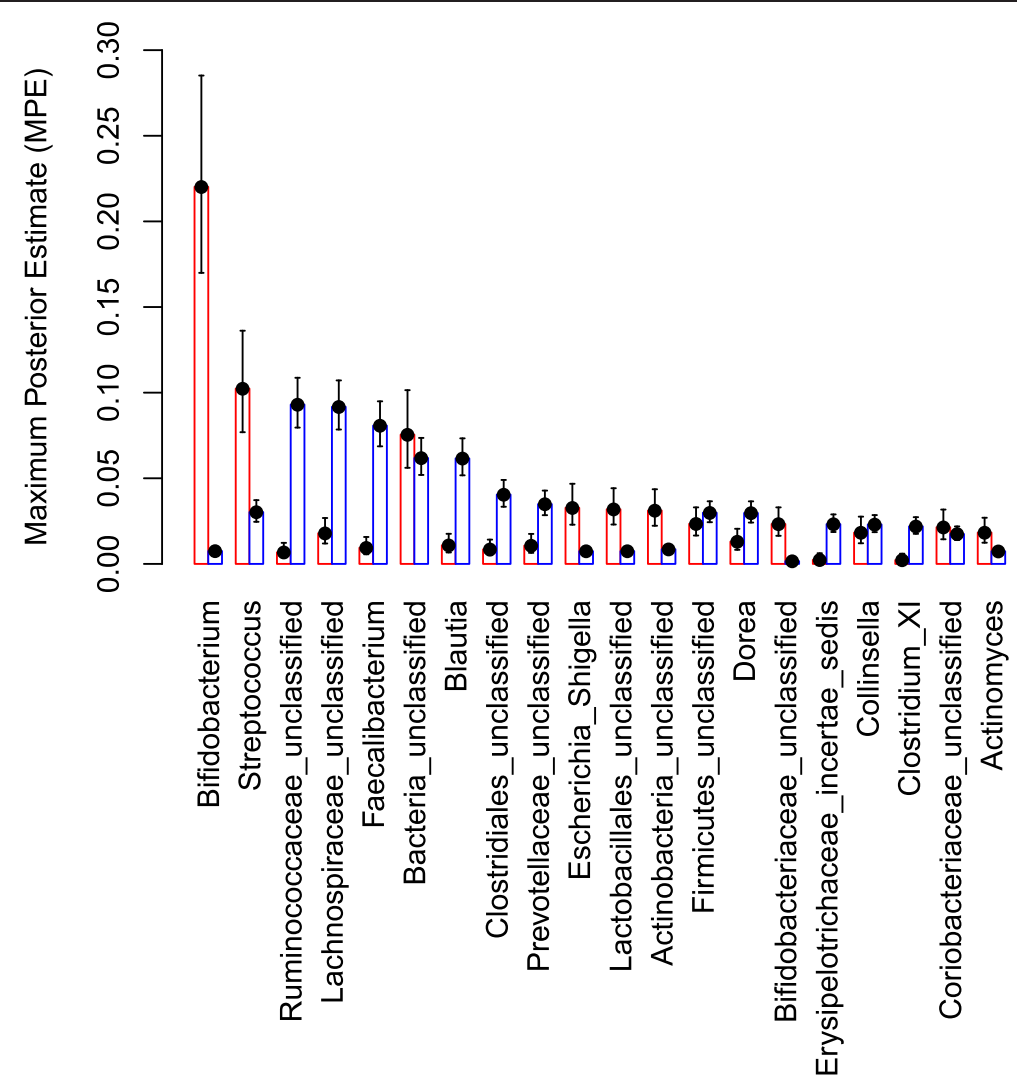

Fig. 8 Mean proportions (Maximum Posterior Estimate) of the twenty most abundant taxa in the two Dirichlet components FM1 (red) and FM2 (blue). The lower and upper $95 \%$ credible intervals are shown as error bars

(Additional file 1: Table S8) revealed a statistically significant increased risk of $P$. falciparum infection for FM2 subjects versus FM1 subjects (hazards ratio $=2.41$; 95 \% CI: 1.33-4.36, $P=0.0038$ ).

Finally, we examined the relationship between the stool microbiota composition present before the malaria season and the prospective risk of developing febrile malaria in subjects who became infected with $P$. falciparum bloodstage parasites during the ensuing malaria season. Again, for this analysis we only included subjects who were PCR negative for $P$. falciparum infection before the malaria season and then became infected with $P$. falciparum during the ensuing malaria season, as detected through bi-weekly PCR of fingerprick blood samples. Malaria immune subjects $(n=49)$ were defined as those whose $P$. falciparum infections never progressed to febrile, symptomatic malaria despite weekly active clinical surveillance; whereas malaria susceptible subjects $(n=63)$ were defined as those whose $P$. falciparum infection progressed to a febrile malaria episode $\left(\mathrm{T} \geq 37.5{ }^{\circ} \mathrm{C}\right.$ and parasite density $\geq 2500$ asexual parasites/ $\mu \mathrm{l}$ ). On the basis of the DMM modeling of the microbiota data described above, the same FM1 and FM2 sample groups had similar proportions of malaria immune and susceptible subjects (FM1: 18 immune and 20 susceptible; FM2: 31 immune and 43 susceptible; $\mathrm{P}=0.55$ ), suggesting that gut microbiota composition is not associated with the risk of febrile malaria once a $P$. falciparum blood-stage infection is established. On the basis of the same Dirichlet sample groups FM1 and FM2, we also generated Kaplan-Meier plots and performed log-rank analysis for time to first febrile malaria episode (Fig. 9b). We found no statistically significant difference between FM1 and FM2 for time to first febrile malaria episode using either the case definition for clinical malaria ( $\mathrm{T} \geq 37.5{ }^{\circ} \mathrm{C}$ and parasite density $\geq 2500$ asexual parasites $/ \mu \mathrm{l})$ or the less restrictive definition of fever and any level of $P$. falciparum parasitemia.

\section{Discussion}

In this study we aimed to describe the composition of the gut microbiota in an age-stratified rural West African population that is exposed to intense seasonal $P$. falciparum transmission. We compared the gut microbiota composition in this population with studies conducted in other regions. In an exploratory analysis we also investigated the relationship between the gut microbiota composition and the prospective risk of $P$. falciparum infection as 

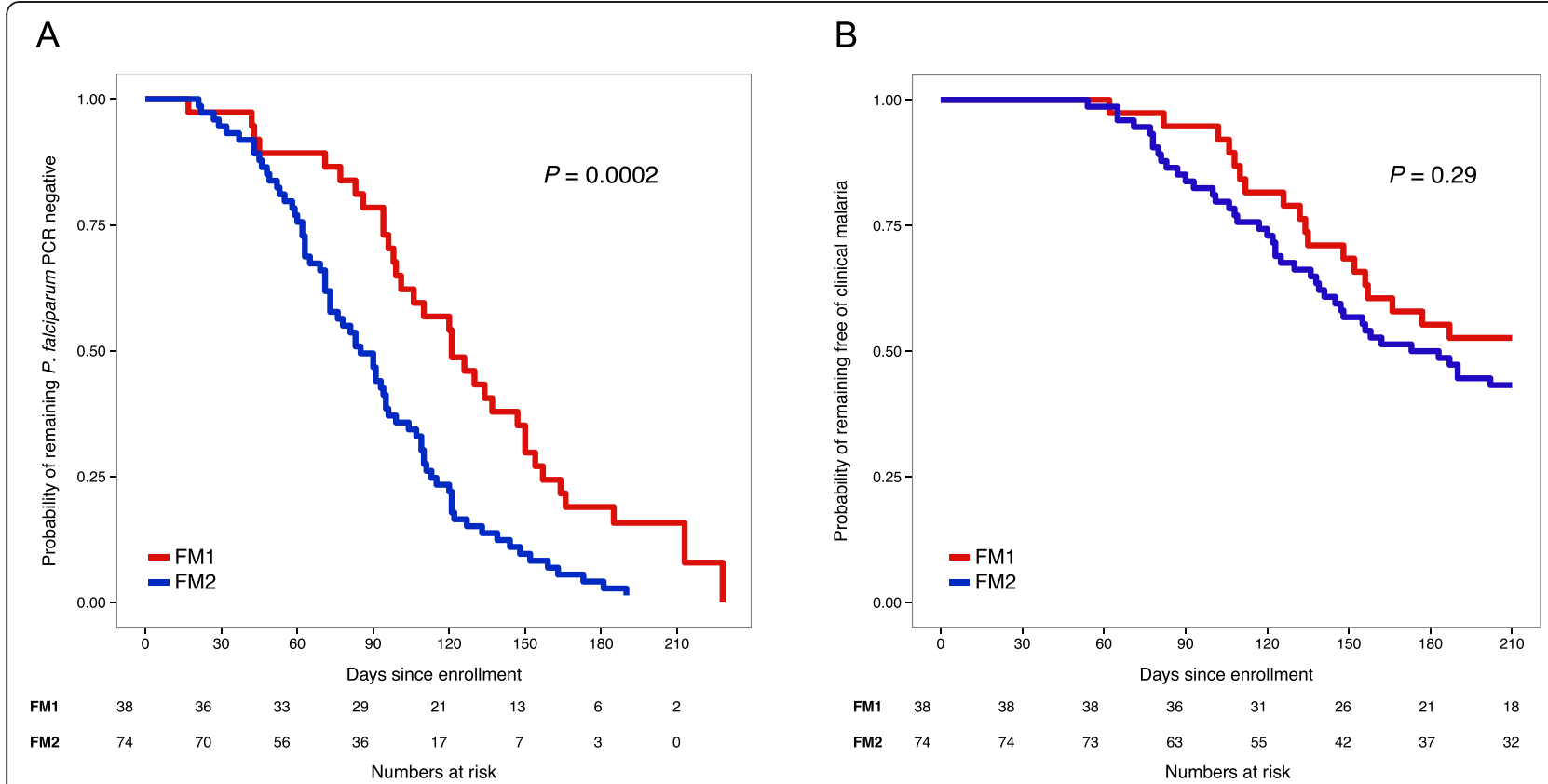

Fig. 9 Stool microbiota composition is associated with the prospective risk of $P$. falciparum infection but not the prospective risk of febrile malaria. Kaplan-Meier plots showing time to (a) the first PCR-confirmed $P$. falciparum blood-stage infection (irrespective of whether symptoms were present) and (b) the first febrile malaria episode among subjects with documented $P$. falciparum infection, stratified by the two DMM sample groups FM1 and FM2. $P$ values comparing FM1 to FM2 were calculated by the log-rank test

well as the risk of developing febrile malaria once P. falciparum infection is established.

Like studies conducted in other regions (for instance, [5]) we found that the alpha diversity of the gut microbiota increased with age in both males and females. We found in this cohort that the most dynamic shifts in microbiota composition occur before the 3-5 years of age, possibly related to the transition from breast milk to solid foods [30] and then changes occur more gradually through adolescence and adulthood. We observed clear differences between the composition of the gut microbiota in this study and that reported in studies conducted in other parts of the world, underscoring the diversity of gut microbiotas across geographic regions. Although the non-uniformity of the $16 \mathrm{~S}$ variable region that was selected for sequencing across these studies may have impacted taxa detection [31], several sample groups shared similar taxa, albeit at different abundance levels. The relative impact of factors underlying the observed variability in microbiota composition across geographic regions such as diet, antibiotic exposure, genetics, and co-infections remains to be determined.

Using a DMM framework we identified two subject groups with distinct microbiota profiles (FM1 and FM2) that exhibited statistically significant differences in the prospective risk of $P$. falciparum infection after accounting for age and other potential confounders. The gut microbiota of subjects at lower risk of $P$. falciparum infection (FM1) contained a significantly higher proportion of Bifidobacterium and Streptococcus compared to subjects at higher risk of $P$. falciparum infection (FM2). This finding raises the possibility that modulation of the microbiota with pre- and/ or pro-biotic nutritional supplementation that promotes the growth of commensal organisms like Bifidobacterium, particularly in the neonatal period [32, 33], may be of benefit in the context of malaria. Importantly, studies indicate that natural components of human milk stimulate the growth of Bifidobacterium [34-36]. Clearly, further studies are needed to understand the potential interactions between specific components of the microbiota, innate/ adaptive immune responses, and susceptibility to malaria and other pathogens that commonly affect this population.

Also of potential interest, the gut microbiota of FM1 subjects contained a significantly higher proportion of the Enterobacteriaceae Escherichia/Shigella compared to subjects at higher risk of P. falciparum infection (FM2) ( $3.2 \%$ versus $0.7 \%$, respectively) - an intriguing finding in light of a recent mouse study in which anti- $\alpha$-gal antibodies induced by the presence of $\alpha$-gal-expressing Escherichia coli in the gut, were cytotoxic to $\alpha$-gal-expressing Plasmodium sporozoites in the skin, thus protecting mice from Plasmodium infection [25]. Because not all Enterobacteriaceae express $\alpha$-gal [37] we are unable to ascertain from the present $16 \mathrm{~S}$ data whether strains in these samples express $\alpha$-gal. A functional screen for the expression of this gene or a metagenomic approach would be required to 
answer this question. It will be of interest in future studies to use higher resolution techniques to determine if differences in the proportion of $\alpha$-gal expressing Enterobacteriaceae are associated with the risk of $P$. falciparum infection. Moreover, additional studies are needed in various malariaendemic areas to determine if the findings here can be replicated, since the association we observed between microbiota composition and $P$. falciparum infection risk may have occurred by chance or may be confounded by other factors not measured in this study.

Importantly, we did not observe a relationship between the gut microbiota composition and the prospective risk of developing febrile malaria once $P$. falciparum blood-stage infections were established, possibly due to a lack of statistical power. However, we observed clear changes in the microbiota composition over the age range during which clinical immunity to febrile malaria is slowly acquired in areas of intense malaria transmission [20], suggesting that age-associated changes in the gut microbiota from infancy to adulthood may play a role in shaping the quality of the immune response to malaria as individuals gradually acquire clinical immunity to malaria, hypotheses that could be further tested in larger longitudinal studies.

\section{Conclusions}

The findings of this study underscore the diversity of the gut microbiota with age and across geographic regions. In addition, the preliminary finding of an association between gut microbiota composition and $P$. falciparum infection risk suggests that strategic modulation of gut microbiota composition could decrease $P$. falciparum infection risk in malaria-endemic areas, potentially as an adjunct to partially effective malaria vaccines.

\section{Methods}

\section{Ethics statement}

The Ethics Committee of the Faculty of Medicine, Pharmacy and Dentistry at the University of Sciences, Technique and Technology of Bamako, the Institutional Review Board (IRB) of the National Institute of Allergy and Infectious Diseases (NIAID), National Institutes of Health (NIH) and of the J. Craig Venter Institute (JCVI) approved this study. Written, informed consent was obtained from adult subjects and from the parents or guardians of participating children.

\section{Study site and subjects}

This cohort study was conducted in Mali in the rural village of Kalifabougou which has a population of 4,394 and is situated $\sim 40 \mathrm{~km}$ northwest of the capital Bamako. Bambara is the predominant ethnic group and $~ 90 \%$ of residents engage in subsistence farming. Kalifabougou is in the savanna ecoclimatic zone where annual rainfall is $800-1,200 \mathrm{~mm} /$ year. Intense transmission of P. falciparum malaria occurs during the rainy season in this region of Mali from June through December. In May 2011, we enrolled 695 healthy individuals aged 3 months to 25 years in a cohort study to investigate mechanisms of naturally acquired immunity to malaria using systems biology approaches. A detailed description of the cohort has been published elsewhere [38]. As part of the initial clinical assessment, spleen size was scored using the Hackett method [39], with splenomegaly defined as a Hackett score $>1$. Baseline hemoglobin values, measured by a HemoCue analyzer, were used to determine anemia status based on WHO criteria, and hemoglobin typing was performed with a D-10 instrument (Bio-Rad) to identify individuals with sickle cell trait (HbAS). Exclusion criteria at enrollment included a hemoglobin level $<7 \mathrm{~g} / \mathrm{dL}$, axillary temperature $\geq 37.5^{\circ} \mathrm{C}$, acute systemic illness, underlying chronic disease, use of antimalarial or immunosuppressive medications in the past 30 days, or pregnancy. As part of a mass drug administration program in Mali [40, 41] all residents greater than 5 years of age received albendazole, ivermectin, and praziquantel in March 2011 (prior to enrollment) and only albendazole and ivermectin in October 2011. For the stool microbiota analysis described here, we randomly selected an age-stratified subset of 200 individuals from the entire cohort; the selection process is shown in Fig. 1.

\section{Surveillance for symptomatic and asymptomatic Plasmodium infection}

After enrollment, individuals were followed during the ensuing malaria season for 7 months. Symptomatic malaria episodes were detected prospectively by self-referral and through weekly active clinical surveillance. All individuals with signs and symptoms of malaria and any level of Plasmodium parasitemia detected by light microscopy were treated according to the National Malaria Control Program guidelines in Mali. Thick blood smears were stained with Giemsa and counted against 300 leukocytes. Parasite densities were recorded as the number of asexual parasites/ $\mu \mathrm{l}$ of blood based on a mean leukocyte count of 7500 cells $/ \mu$ l. Each smear was read in a blinded manner by two certified microscopists. The research definition of clinical malaria was parasitemia of $\geq 2500$ parasites $/ \mu \mathrm{L}$, an axillary temperature of $\geq 37.5^{\circ} \mathrm{C}$ within $24 \mathrm{~h}$, and no other cause of fever discernible by physical exam. To detect asymptomatic Plasmodium infections during the 7-month study period, blood was collected by fingerprick onto filter paper at bi-weekly scheduled visits. At the end of the study period, stored filter papers were analyzed by PCR chronologically from enrollment onwards until the first $P$. falciparum infection of the malaria season was 
detected for each individual. A detailed description of the PCR protocol was published elsewhere [20].

\section{Detection of urinary and intestinal parasites}

Stool and urine samples were collected at enrollment. $S$. haematobium eggs were quantified by microscopy after filtration of fresh urine samples with Nytrel filters (Vestergaard Frandsen). Schistosoma mansoni and other geohelminth eggs were detected by microscopy of duplicate fresh fecal thick smears using the Kato-Katz method [42]. Aliquots of stool were cryopreserved at $-80{ }^{\circ} \mathrm{C}$ for DNA extraction and multi-parallel, real-time PCR for intestinal nematodes (Necator americanus, Ancylostoma duodenale, Trichuris trichiura, Ascaris lumbricoides, and Strongyloides stercoralis) was performed as previously described [43]. Individuals diagnosed with urinary schistosomiasis were treated with praziquantel (see Table 1).

\section{Stool collection and microbial DNA extraction}

The sub-cohort that is the primary focus of this study was comprised of 200 subjects-106 females and 94 males ranging in age from 3 months to 25 years. Stool samples were collected at study enrollment in May 2011 just prior to the six-month malaria season. Stool samples were stored in $-80{ }^{\circ} \mathrm{C}$ freezers in Mali and shipped to the U.S. on dry ice for analysis. Microbial DNA for $16 \mathrm{~S}$ rDNA gene sequencing was isolated from stool samples using enzymatic lysis followed by phenol-chloroform isoamyl alcohol extraction and ethanol precipitation. Standard Roche protocols were used for sample preparation.

\section{$16 \mathrm{~S}$ rRNA gene sequencing}

The 16S rRNA gene amplicons were quantified and pooled in equal mass ratios such that amplicons derived from samples to be analyzed could be multiplexed in a single Roche-454 sequencing run. Established 454 FLX sequencing methods (Roche) that were developed for the HMP, including universal PCR primers with unique barcode identifiers, were used to amplify and sequence the hypervariable regions (V1-V3) of the bacterial $16 \mathrm{~S}$ rRNA gene [31].

\section{Comparative dataset analysis}

16S rRNA gene sequence data generated from stool samples collected as part of three other studies were included in the analysis component of this study for comparative purposes only. These additional studies included a cohort in Malawi [5], the HMP cohort [44], and the healthy controls from the moderate-to-severe diarrhea (MSD) study [6]. Data for the Malawi cohort was retrieved from MGRAST (http://metagenomics.anl.gov/). The Malawi cohort was part of accession "mgp401" (http://metagenomics.anl. gov/linkin.cgi?project=401) [5]. This study sequenced the
V4 region of the 16S rRNA gene using Illumina HiSeq 2000. We were primarily interested in the Malawi cohort because of similarities in age distribution and geographic location. Specifically, the Malawi cohort was comprised of 114 subjects ranging in age from 1.5 weeks to 44 years (age data for 31 subjects were unavailable) and consisted of 63 females and 20 males (gender data for the remaining subjects were unavailable). In common with the present Mali cohort, sequences from the HMP cohort were derived from the V1-V3 regions of the stool bacterial 16S rRNA gene using 454 pyrosequencing. These samples originated from 140 stool samples collected and processed during the HMP. Raw sequence data and metadata were retrieved from the HMP Data Analysis and Coordination Center (HMPDACC) (http://www.hmpdacc.org/). The subjects in the HMP cohort ranged in age from 18 to 40 years and 73 were females and 67 males. Data for the MSD study were downloaded from SRA at NCBI and were available under the bioproject accession PRJNA234437. In the MSD study sequence data was generated (using 454 FLX sequencing) from the V1-V2 region of the $16 \mathrm{~S}$ rRNA gene from DNA extracted from stool samples. Healthy control samples in the MSD study were identified from the full data set using the available study meta-data and only these (493 in total) were included in the comparison here. The healthy control samples in the MSD study were collected from individuals residing in Bangladesh (117), The Gambia (134), Kenya (140) and Mali (102). These individuals were all under 5 years of age (ranging from 1 to 58 months) and no gender information was available from the study metadata.

\section{$16 \mathrm{~S}$ rRNA sequence data processing}

For the 454 data, the raw flowgram files (Standard Flowgram Format) were deconvolved using the sample barcode information, and the barcodes and primers were trimmed subsequently. All processing of the 454 and Illumina sequences from the various studies were carried out using mothur v1.28 [45] - quality check, sequence screening and filtering, chimera checking, and taxonomy assignment steps used the HMP SOP for $16 \mathrm{~S}$ rRNA gene (16S) curation (http://www.hmpdacc.org/ HM16STR/). OTUs at $97 \%$ sequence identity were computed for the Malian cohort in order to assess subgenus diversity and structure. The comparisons of the present Malian cohort to the other three datasets were carried out only at the genus level.

For the Malian cohort, from the initial 200 samples, 195 samples were successfully sequenced and each sample yielded more than 1,000 16S rRNA sequences after barcode and primer trimming. Together, the samples resulted in 1,500,243 sequences that were used for downstream analysis. Reads were aligned against a reference template, trimmed (keeping only $>=100 \mathrm{bp}$ sequences), and checked for chimeras. The resulting output consisted 
of 1,331,167 sequences. These were assigned taxonomy down to the genus level using mothur's implementation of the RDP classifier (using a bootstrap confidence threshold of $80 \%$ ). For OTU analysis, this sequence set was processed further by alignment column trimming, in which the required length of trimmed sequences was $>=200 \mathrm{bp}$. This yielded 968,362 total sequences. These were clustered at $97 \%$ sequence identity to generate OTUs.

\section{Statistical analysis of the $16 \mathrm{~S}$ data}

The taxonomic assignments of the $16 \mathrm{~S}$ sequences were used to generate sample-taxa count matrices. These matrices were analyzed using a DMM modeling framework [28] to identify sample groups, where each sample group (also known as a Dirichlet component or cluster) can be summarized by a vector of taxa proportions. The optimal number of Dirichlet components along with the mean proportion of each taxa in a Dirichlet component was calculated using the software developed by Holmes et al. [28]. The program was run for various values of $K$, the number of components, and subsequently, the $K$ with the best model fit using the Laplace approximation to the negative log model evidence was chosen as the optimal value for that dataset. The Dirichlet component means were calculated from the Maximum Posterior Estimates (MPE) of the model hyperparameters [28].

\section{Statistical analysis of the clinical data}

For continuous variables we compared the differences between group medians with the Kruskal-Wallis test (Table 2); for binary variables, group comparisons were performed using Fisher's exact test. Kaplan-Meier curves were used to estimate the probability of remaining free of clinical malaria. For individuals who began the malaria season PCR-negative for Plasmodium, Kaplan-Meier curves were also used to estimate the probability of remaining free of blood-stage $P$. falciparum infection. Individuals who were infected with Plasmodium malariae were censored at the time of infection. Log-rank analysis was used to test the significance of differences in time to first febrile malaria episode and time to first P. falciparum infection between DMM clusters. A Cox proportional hazards model was used to estimate the risk of $P$. falciparum infection between DMM clusters and included the following potentially confounding variables: age (per one year increase), HbAS, gender, mild anemia at enrollment, $S$. haematobium infection, presence of splenomegaly at enrollment (Hackett score $>1$ ) and distance from home to river (largest stream in Kalifabougou; per $100 \mathrm{~m}$ increase). This combination of variables met the assumptions of proportional hazards. Of these variables, HbAS has been shown to affect the risk of $P$. falciparum infection [46, 47], and we have evidence to suggest that baseline splenomegaly increases subsequent to $P$. falciparum infection risk (unpublished data). Statistical significance was defined as a 2 -tailed $P$ value of $<0.05$. We performed all analyses in $\mathrm{R}$ version 3.1.1 (http://www.R-project.org).

\section{Availability of supporting data}

The data sets supporting the results of this article are included within the article (and its additional file(s)). The sequence data for the Malian cohort are available at NCBI's Sequence Read Archive under BioProject PRJNA285808. These data are also available at MGRAST under Project 4793.

\section{Additional file}

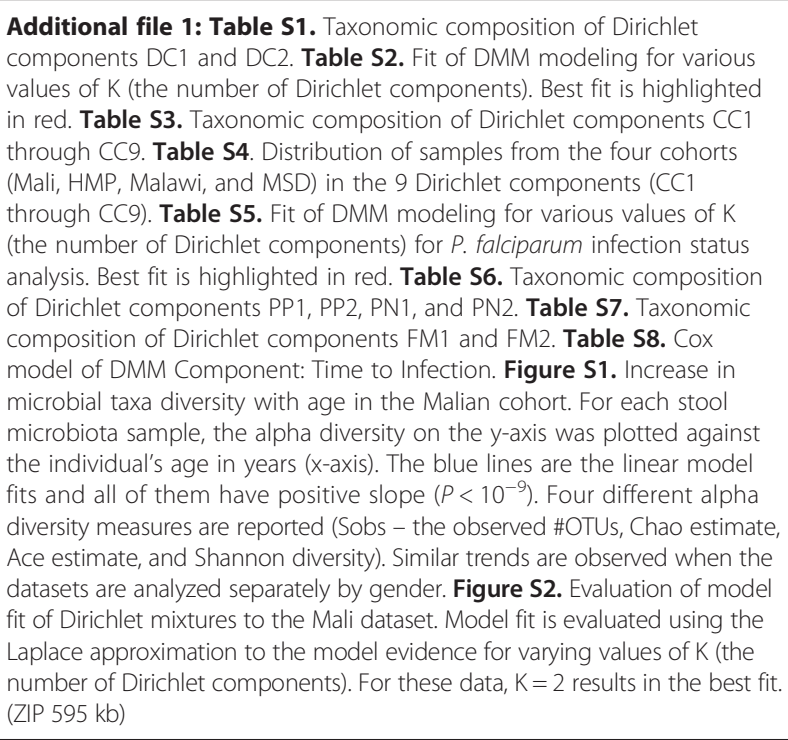

\section{Abbreviations}

HMP: Human Microbiome Project; PCR: Polymerase Chain Reaction; rRNA: ribosomal ribonucleic acid; MSD: Moderate-to-severe diarrhea; DMM: Dirichlet Multinomial Mixture; MPE: Maximum Posterior Estimates.

\section{Competing interests}

The authors declare that they have no competing interests.

\section{Author's contributions}

Conceived and designed the experiments: KEN, PDC; Performed the experiments: MGT, MBJ, EOC; Analyzed the data: SY, EFK, TMT, DMH, PDC, KEN; Contributed reagents/materials/analysis tools: SY, EFK, TMT, DMH, MBJ, TBN, SD, OKD, BT, PDC, KEN; Wrote the paper: SY, EFK, TMT, DMH, PDC, KEN. All authors read and approved the final manuscript.

\section{Acknowledgments}

This project was funded with federal funds from the National Institute of Allergy and Infectious Diseases, National Institutes of Health, Department of Health and Human Services, under contract number HHSN272200900007C (PI: Karen E. Nelson). The cohort study in Mali was supported by the Division of Intramural Research, National Institute of Allergy and Infectious Diseases, National Institutes of Health, under 1 ZIA Al001126 (PI: Peter D. Crompton).

\section{Author details}

1J. Craig Venter Institute, 4120 Capricorn Lane, La Jolla, CA 92037, USA. ${ }^{2}$ J. Craig Venter Institute, 9704 Medical Center Drive, Rockville, MD 20850, USA. ${ }^{3}$ Laboratory of Immunogenetics, Division of Intramural Research, National Institute of Allergy and Infectious Diseases, National Institutes of Health, 
Rockville, MD 20852, USA. ${ }^{4}$ Laboratory of Parasitic Diseases, Division of Intramural Research, National Institute of Allergy and Infectious Diseases, National Institutes of Health, Bethesda, MD 20892, USA. ${ }^{5}$ Malaria Research and Training Centre, Department of Epidemiology of Parasitic Diseases, International Center of Excellence in Research, University of Sciences, Technique and Technology of Bamako, Bamako, Mali.

\section{Received: 22 April 2015 Accepted: 5 August 2015} Published online: 22 August 2015

\section{References}

1. Gill SR, Pop M, Deboy RT, Eckburg PB, Turnbaugh PJ, Samuel BS, et al. Metagenomic analysis of the human distal gut microbiome. Science. 2006;312(5778):1355-9.

2. Human Microbiome Project C. Structure, function and diversity of the healthy human microbiome. Nature. 2012;486(7402):207-14.

3. Human Microbiome Project C. A framework for human microbiome research. Nature. 2012;486(7402):215-21.

4. Qin J, Li R, Raes J, Arumugam M, Burgdorf KS, Manichanh C, et al. A human gut microbial gene catalogue established by metagenomic sequencing. Nature. 2010;464(7285):59-65

5. Yatsunenko T, Rey FE, Manary MJ, Trehan I, Dominguez-Bello MG, Contreras $M$, et al. Human gut microbiome viewed across age and geography. Nature. 2012;486(7402):222-7.

6. Pop M, Walker AW, Paulson J, Lindsay B, Antonio M, Hossain M, et al. Diarrhea in young children from low-income countries leads to large-scale alterations in intestinal microbiota composition. Genome biology. 2014;15(6):R76.

7. Subramanian S, Huq S, Yatsunenko T, Haque R, Mahfuz M, Alam MA, et al. Persistent gut microbiota immaturity in malnourished Bangladeshi children. Nature. 2014;510(7505):417-21.

8. Cotillard A, Kennedy SP, Kong LC, Prifti E, Pons N, Le Chatelier E, et al. Dietary intervention impact on gut microbial gene richness. Nature. 2013;500(7464):585-8.

9. David LA, Maurice CF, Carmody RN, Gootenberg DB, Button JE, Wolfe BE, et al. Diet rapidly and reproducibly alters the human gut microbiome. Nature. 2014;505(7484):559-63.

10. Kamada N, Chen GY, Inohara N, Nunez G. Control of pathogens and pathobionts by the gut microbiota. Nature immunology. 2013;14(7):685-90.

11. Uematsu S, Fujimoto $K$, Jang $M H_{1}$, Yang BG, Jung YJ, Nishiyama $M$, et al. Regulation of humoral and cellular gut immunity by lamina propria dendritic cells expressing Toll-like receptor 5. Nature immunology. 2008;9(7):769-76.

12. Hall JA, Bouladoux N, Sun CM, Wohlfert EA, Blank RB, Zhu Q, et al. Commensal DNA limits regulatory $T$ cell conversion and is a natural adjuvant of intestinal immune responses. Immunity. 2008;29(4):637-49.

13. Shulzhenko N, Morgun A, Hsiao W, Battle M, Yao M, Gavrilova O, et al. Crosstalk between B lymphocytes, microbiota and the intestinal epithelium governs immunity versus metabolism in the gut. Nature medicine. 2011;17(12):1585-93.

14. Mazmanian SK, Liu CH, Tzianabos AO, Kasper DL. An immunomodulatory molecule of symbiotic bacteria directs maturation of the host immune system. Cell. 2005;122(1):107-18.

15. Clarke TB, Davis KM, Lysenko ES, Zhou AY, Yu Y, Weiser JN. Recognition of peptidoglycan from the microbiota by Nod1 enhances systemic innate immunity. Nature medicine. 2010;16(2):228-31.

16. Ivanov II WHJ, Darce J, Hattori K, Shima T, Umesaki Y, Littman DR, et al. Gut-residing segmented filamentous bacteria drive autoimmune arthritis via T helper 17 cells. Immunity. 2010;32(6):815-27.

17. WHO World Malaria Report 2014 [http://www.who.int/malaria/publications/ world_malaria_report_2014/en/]

18. Marsh K, Kinyanjui S. Immune effector mechanisms in malaria. Parasite immunology. 2006;28(1-2):51-60.

19. Langhorne J, Ndungu FM, Sponaas AM, Marsh K. Immunity to malaria: more questions than answers. Nature immunology. 2008;9(7):725-32.

20. Tran TM, Li S, Doumbo S, Doumtabe D, Huang CY, Dia S, et al. An intensive longitudinal cohort study of Malian children and adults reveals no evidence of acquired immunity to Plasmodium falciparum infection. Clin Infect Dis. 2013;57(1):40-7

21. Crompton PD, Moebius J, Portugal S, Waisberg M, Hart G, Garver LS, et al. Malaria immunity in man and mosquito: insights into unsolved mysteries of a deadly infectious disease. Annu Rev Immunol. 2014;32:157-87.
22. Goncalves BP, Huang CY, Morrison R, Holte S, Kabyemela E, Prevots DR, et al. Parasite burden and severity of malaria in Tanzanian children. New Engl J Med. 2014;370(19):1799-808.

23. Kwiatkowski DP. How malaria has affected the human genome and what human genetics can teach us about malaria. Am J Hum Gene. 2005;77(2):171-92.

24. Turner L, Lavstsen T, Berger SS, Wang CW, Petersen JE, Avril M, et al. Severe malaria is associated with parasite binding to endothelial protein $C$ receptor. Nature. 2013:498(7455):502-5.

25. Yilmaz B, Portugal S, Tran TM, Gozzelino R, Ramos S, Gomes J, et al. Gut Microbiota Elicits a Protective Immune Response against Malaria Transmission. Cell. 2014;159(6):1277-89.

26. Li M, Wang B, Zhang M, Rantalainen M, Wang S, Zhou H, et al. Symbiotic gut microbes modulate human metabolic phenotypes. Proc Natl Acad Sci U S A. 2008;105(6):2117-22.

27. Riley EM, Wagner GE, Akanmori BD, Koram KA. Do maternally acquired antibodies protect infants from malaria infection? Parasite Immunol. 2001;23(2):51-9.

28. Holmes I, Harris K, Quince C. Dirichlet multinomial mixtures: generative models for microbial metagenomics. PloS one. 2012;7(2):e30126.

29. Weiss GE, Traore B, Kayentao K, Ongoiba A, Doumbo S, Doumtabe D, et al. The Plasmodium falciparum-specific human memory B cell compartment expands gradually with repeated malaria infections. PLoS pathogens. 2010;6(5):e1000912.

30. Palmer C, Bik EM, DiGiulio DB, Relman DA, Brown PO. Development of the human infant intestinal microbiota. PLoS Biol. 2007;5(7):e177.

31. Jumpstart Consortium Human Microbiome Project Data Generation Working G. Evaluation of 165 rDNA-based community profiling for human microbiome research. PloS one. 2012;7(6):e39315

32. Chassard C, de Wouters T, Lacroix C. Probiotics tailored to the infant: a window of opportunity. Curr Opin Biotechnol. 2014;26:141-7.

33. Gritz EC, Bhandari V. The human neonatal gut microbiome: a brief review. Frontiers in pediatrics. 2015;3:17.

34. Jain N, Walker WA. Diet and host-microbial crosstalk in postnatal intestinal immune homeostasis. Nat Rev Gastroenterol Hepatol. 2015;12(1):14-25.

35. Oozeer R, van Limpt K, Ludwig T, Ben Amor K, Martin R, Wind RD, et al. Intestinal microbiology in early life: specific prebiotics can have similar functionalities as human-milk oligosaccharides. Am J Clin Nutr. 2013;98(2):561S-71.

36. Sela DA, Mills DA. Nursing our microbiota: molecular linkages between bifidobacteria and milk oligosaccharides. Trends Microbiol. 2010;18(7):298-307.

37. Galili U, Mandrell RE, Hamadeh RM, Shohet SB, Griffiss JM. Interaction between human natural anti-alpha-galactosyl immunoglobulin $\mathrm{G}$ and bacteria of the human flora. Infect Immun. 1988;56(7):1730-7.

38. Doumbo S, Tran TM, Sangala J, Li S, Doumtabe D, Kone Y, et al. Co-infection of long-term carriers of Plasmodium falciparum with Schistosoma haematobium enhances protection from febrile malaria: a prospective cohort study in Mali. PLoS neglected tropical diseases. 2014;8(9):e3154

39. Baird JK, Bangs MJ, Maguire JD, Barcus MJ. Epidemiological measures of risk of malaria. Meth Mol Med. 2002;72:13-22.

40. Dembele M, Bamani S, Dembele R, Traore MO, Goita S, Traore MN, et al. Implementing preventive chemotherapy through an integrated National Neglected Tropical Disease Control Program in Mali. PLoS neglected tropical diseases. 2012;6(3):e1574.

41. Landoure A, Dembele R, Goita S, Kane M, Tuinsma M, Sacko M, et al. Significantly reduced intensity of infection but persistent prevalence of schistosomiasis in a highly endemic region in Mali after repeated treatment. PLOS neglected tropical diseases. 2012;6(7):e1774.

42. Katz N, Chaves A, Pellegrino J. A simple device for quantitative stool thicksmear technique in Schistosomiasis mansoni. Revista do Instituto de Medicina Tropical de Sao Paulo. 1972;14(6):397-400.

43. Mejia R, Vicuna Y, Broncano N, Sandoval C, Vaca M, Chico M, et al. A novel, multi-parallel, real-time polymerase chain reaction approach for eight gastrointestinal parasites provides improved diagnostic capabilities to resource-limited at-risk populations. Am J Trop Med Hyg. 2013;88(6):1041-7.

44. Gevers D, Knight R, Petrosino JF, Huang K, McGuire AL, Birren BW, et al. The human microbiome project: a community resource for the healthy human microbiome. PLoS Biol. 2012;10(8):e1001377.

45. Schloss PD, Westcott SL, Ryabin T, Hall JR, Hartmann M, Hollister EB, et al. Introducing mothur: open-source, platform-independent, communitysupported software for describing and comparing microbial communities. Appl Environ Microbiol. 2009;75(23):7537-41 
46. Gong L, Maiteki-Sebuguzi C, Rosenthal PJ, Hubbard AE, Drakeley CJ, Dorsey $G$, et al. Evidence for both innate and acquired mechanisms of protection from Plasmodium falciparum in children with sickle cell trait. Blood. 2012;119(16):3808-14.

47. Mangano VD, Kabore Y, Bougouma EC, Verra F, Sepulveda N, Bisseye C,

et al. Novel insights into the protective role of haemoglobin $S$ and $C$

against Plasmodium falciparum parasitaemia. J Infect Dis. 2015;212(4):626-34.

\section{Submit your next manuscript to BioMed Central} and take full advantage of:

- Convenient online submission

- Thorough peer review

- No space constraints or color figure charges

- Immediate publication on acceptance

- Inclusion in PubMed, CAS, Scopus and Google Scholar

- Research which is freely available for redistribution 